\title{
Performance Reduction for Automatic Development of Parallel Applications for Reconfigurable Computer Systems
}

\author{
Aleksey I. Dordopulo ${ }^{1}$, Ilya I. Levin ${ }^{2}$ \\ (C) The Authors 2020. This paper is published with open access at SuperFri.org
}

\begin{abstract}
In the paper, we review a suboptimal methodology of mapping of a task information graph on the architecture of a reconfigurable computer system. Using performance reduction methods, we can solve computational problems which need hardware costs exceeding the available hardware resource. We proved theorems, concerning properties of sequential reductions. In our case, we have the following types of reduction such as the reduction by number of basic subgraphs, by number of computing devices, and by data width. On the base of the proved theorems and corollaries, we developed the methodology of reduction transformations of a task information graph for its automatic adaptation to the architecture of a reconfigurable computer system. We estimated the maximum number of transformations, which, according to the suggested methodology, are needed for balanced reduction of the performance and hardware costs of applications for reconfigurable computer systems.

Keywords: performance reduction, hardware costs, reconfigurable computer system, parallel applications development, information graph.
\end{abstract}

\section{Introduction}

Most researchers of parallel computing [1 4] admit that parallel programming is a complex area. It is necessary to organize and control a large number of processes that asynchronously run on the nodes of a multiprocessor computer system (MCS). The demanding requirements are decreasing of the calculation time and increasing of the results accuracy. To fulfill these requirements, we increase the number of nodes of a multiprocessor computer system, but at the same time, development of parallel programs becomes more complex.

For a long time, we believed that it is possible to cope with the growing complexity of parallel program development with the help of automatic parallelization of sequential processor (procedural) programs. In this case, a parallelizing compiler 1, $1,2,5,10$ receives an imperative processor program, reconstructs the natural parallel structure of its initial algorithm, detects its fragments for concurrent execution (e.g. loop iterations suitable for parallelization), and adds all necessary instructions. However, the automatic parallelization of sequential programs is a computationally expensive problem with an extremely large number of variants for analysis.

The parallelizing compiler has to analyze different variants of multiple fragments of the procedural program. At the same time, it analyses distribution of data among the nodes of the multiprocessor computer system according to its switching network. These two reasons complicate automatic parallelization for clusters that are the most widely used multiprocessor computer systems with distributed memory.

Let us have a cluster computer system, which consists of $n$ nodes, and each node processes its local part of data. In this case, we describe the data distribution among the nodes using an $n$-ary tree. According to the Cayley theorem, we estimate the number of variants of data distribution as the number of different trees for $n$ vertices, i.e. $n^{n-2}$. For example, the cluster MCS consists of 64 nodes. So, the number of possible distribution variants is $64^{62}=2^{372}$. Analysis of such number of variants on any existing computer system and during any reasonable time is impossible.

\footnotetext{
${ }^{1}$ Supercomputers and Neurocomputers Research Center, Taganrog, Russia

${ }^{2}$ Southern Federal University, Taganrog, Russia
} 
Therefore, the most part of research in this problem domain was devoted to heuristic methods of search space reduction (e.g. the analysis of information dependencies [1], loop nests and iterations [4], private and reduction variables 7.8. loop fusion, loop distribution 9, 10, etc.). Formal transformations and heuristic methods, developed for rejection of inefficient parallel program variants [3], require some recommendations and instructions given by the programmer; otherwise, they cannot provide efficient automatic parallelization of any procedural program.

Nowadays, multi-chip reconfigurable computer systems (RCS) [11] with field-programmable gate arrays (FPGAs) are widely used for solving of computationally expensive problems in various fields of science and technology. RCSs contain multiple FPGAs of a large logical capacity. The FPGAs are connected by a spatial switching system into a single computational field. Within such computational field, we implement calculations as a computing structure 12 14 and decrease the solution time 15, 16, by one or two orders of magnitude at the considerably lower (by a factor of 6-8) processing rate. For certain problem domains [17, 18], RCSs are considerably superior in real performance and power efficiency in contrast with cluster MCSs.

In the paper, we consider a theory which helps to reduce the number of variants parallel calculations for analysis and further synthesis of a computing structure for an RCS. We represent a task as an information graph and then, using performance reduction methods and a relatively small number of steps, we transform it into the form, similar to the architecture of an RCS. For most applications, it is possible to synthesize computing structures and to increase the task solution time owing to the performance reduction methods. In this case, the efficiency of the designed structures is not less than $50 \%$ in comparison with those designed by circuit engineers.

Let us review the structure of the paper. In the first section we describe the forms of parallel calculations, and the task information graph used for structural and procedural calculations on the RCS. In the second section, we consider performance reduction as a way of implementing of the task information graph on the RCS with the lack of its hardware resource. In the third section, we represent the performance reduction methods for decreasing of hardware costs, required for implementing of the information graph, and prove theorems on the applicability of reduction transformations. In the fourth section, we represent the performance reduction principles for mapping of the task information graph on the RCS architecture. Besides, here we estimate the number of computing structures that are to be analysed for adaptation of the initial task information graph to the architecture and hardware resource of the RCS. In the fifth section, we describe the rules, according to which we use the reduction transformations in an experiment for verification of our performance reduction methods. The rules were used in tools for parallel application development. In the conclusion we generalize our results and discuss the directions of our future research.

\section{Forms of Calculations}

According to the form of calculations, we can reveal the natural parallelism of the task 1,19$]$. As a result, it simplifies the task solution and scaling. Usually, parallel calculations are represented in two most common forms - an algorithmic diagram (a flowchart) and a graph [1, 19]. According to the algorithmic diagram [1], calculations are a control transfer among computing devices. Besides, the algorithmic diagram defines the order (or the sequence) of operations performed by a computing device (or devices) using processor instructions. Generally, the algorithmic diagram is the task flowchart or pseudocode, but sometimes it is a control flow graph [1,5, 8 
When we represent calculations as a graph, we describe a task or its fragment in an absolutely parallel form, i.e. as an acyclic oriented graph with input, output, and operation vertices connected by arcs according to the data processing order (but not according to the control transfer). There are various forms of graph models for computational tasks such as algorithm graphs [1], information graphs 1, 19], dependency and influence graphs, and lattice graphs [1]. Arcs of a graph show, how arguments of operation vertices depend on results of calculations, performed by other operation vertices, or arguments, received from input vertices. This is an information connection (or information dependence) that describes relations between two vertices of a graph when the output argument of each vertex is the input of another one. If we speak about multiprocessor architectures [1], then information dependence between two operators means addressing to the same memory cell during their execution. If we speak about dataflow architectures, then it means addressing to one and the same element of a flow.

The most common forms are an algorithm graph [1] and an information graph [1, 19]. The algorithm graph describes a computational task as a set of simple operations (addition, multiplication, division, etc.) distributed into levels. Although, it is possible to use complex composite operations (macro operations) as level vertices [1]. All vertices of the algorithm graph, represented in the canonical parallel form, are distributed into numbered subsets, which form levels. Here, the first vertex of each arc belongs to the level, whose number is less than the number of the level, which contains the last vertex. Besides, arcs cannot connect vertices which belong to the same level.

The theory of structural and procedural calculations [19] deals with a task information graph (TIG). In contrast to the parallel forms of an algorithm graph, the task information graph is a combination of layers and iterations. A layer consists of isomorphic, functionally complete, and information independent subgraphs of a task instead of operation vertices. Iterations describe dependencies among processing data over time without considering latency. Subgraphs from one and the same layer are information independent, i.e. not connected by arcs. Subgraphs, which belong to different iterations, depend on processing data. The number of isomorphic subgraphs in a layer is similar to the level width of the canonical parallel form, and the number of iterations is similar to its height, if we consider isomorphic subgraphs as macro-operations. In comparison with an algorithm graph, a TIG describes a task at a higher level of hierarchy. In this case, we use separate operations, but subgraphs which consist of several operations. The information graph describes the absolutely parallel form of the task. The task parameters define the number of iterations. Therefore, the TIG has no dataflows.

A structural implementation of a TIG on a computer system provides the highest performance. In this case, the number of devices is equal to the number of operation vertices (or operations) of a solving task, and the number of input/output arcs is equal to the number of external memory channels. For the majority of applications, such structural implementation of a TIG is impossible, because the number of devices and channels in any RCS is limited. Therefore, if we map a TIG on a real RCS with its limited hardware resource, we transform this TIG into a computing structure with the lower performance and lower requirements for the number of channels, the number of concurrently functioning devices, and/or the data width in comparison with the structural implementation of this TIG.

A TIG (or its subgraph) describes the logical structure of calculations as vertices and arcs. To implement the TIG on an RCS means to create its computing structure, which consists of hardware-programmed devices with timing characteristics such as latency, data processing 


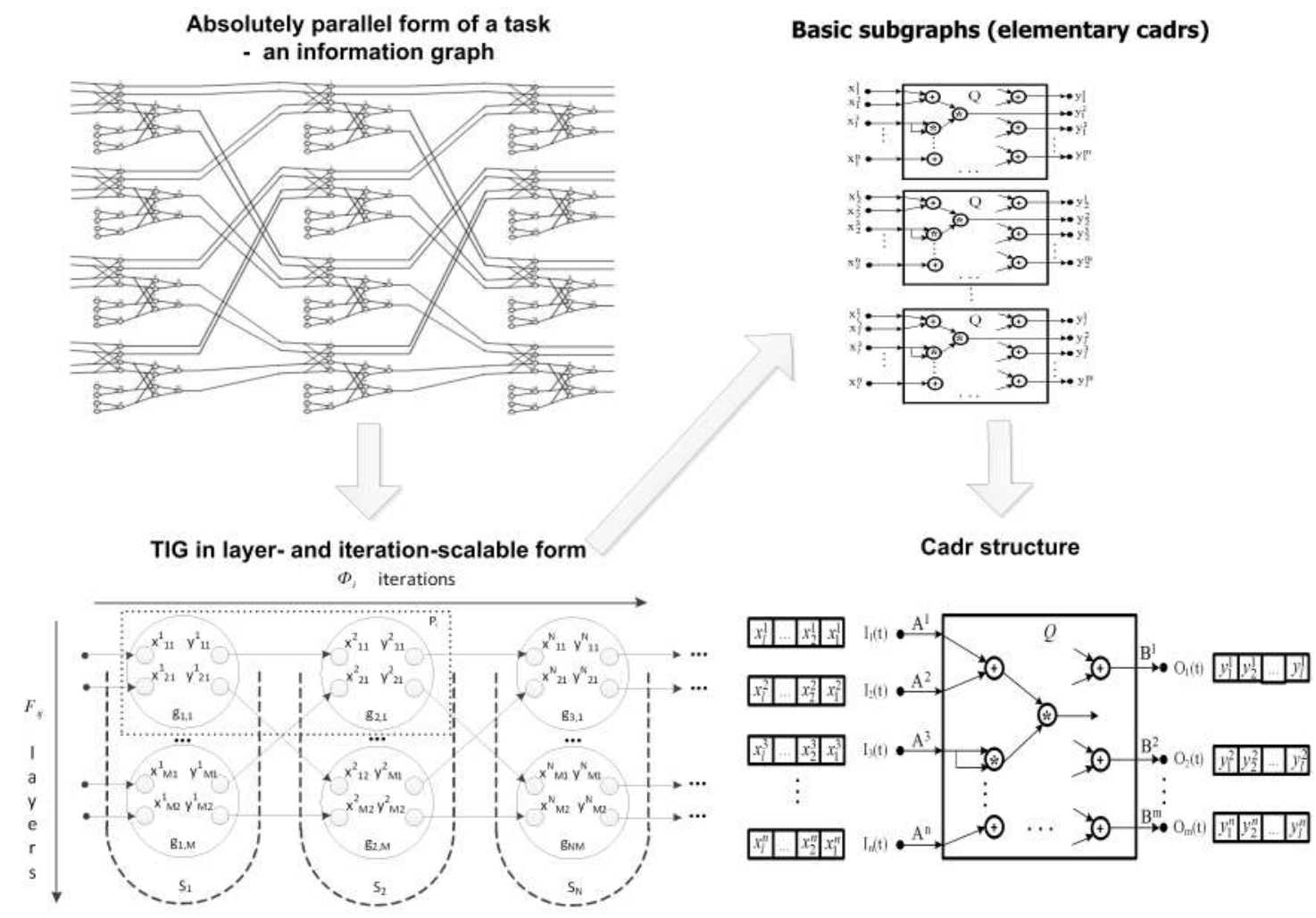

Figure 1. Transformation of a TIG into the computational structure via layer- and iterationscalable form

interval, clock rate, etc. We assume, that the term "implementation of a subgraph on a computer system" means a computing structure which consists of hardware-programmed devices with timing characteristics (or so-called timing component).

Figure 1 shows the transformation a TIG for its structural implementation on an RCS.

To transform the absolutely parallel form of a TIG into the layer- and iteration-scalable form, we obtain the functionally regular form [19] with functions of layer mapping between iterations $\Phi_{i}$, and functions of isomorphic subgraph ordering in a layer $F_{i j}$ :

$$
G=\Phi\left(F_{i j}\left(g_{i j}\right)\right)
$$

where $g_{i j}$ is a basic subgraph (a pipeline computing structure); $F_{i j}$ is an ordering function for information-independent subgraphs in a computational layer; $\Phi_{i}$ is a mapping function of information-dependent layers. The composition of functions $F_{i j}$ and $\Phi_{i}$ depends on an available RCS hardware resource $A_{R C S}$.

Figure 2 shows the task information graph, which consists of information-dependent layers $S_{1}, \ldots, S_{N}$. Each layer consists of isomorphic information-independent subgraphs $G_{1,1}, \ldots, G_{1, M}, \ldots G_{N, M}$.

Owing to such form, we easily scale the task computing structure. If we change the number of basic subgraphs $g_{i j}$ in the composition of the functions $F_{i j}$ and $\Phi_{i}$, then we scale the computing structure both by layers and by iterations. If we increase the number of hardware-programmed information-independent subgraphs within the layer, then we scale the computing structure by layers. If we increase the number of hardware-programmed subgraphs with information dependence among iterations, then we scale the computing structure by iterations. 


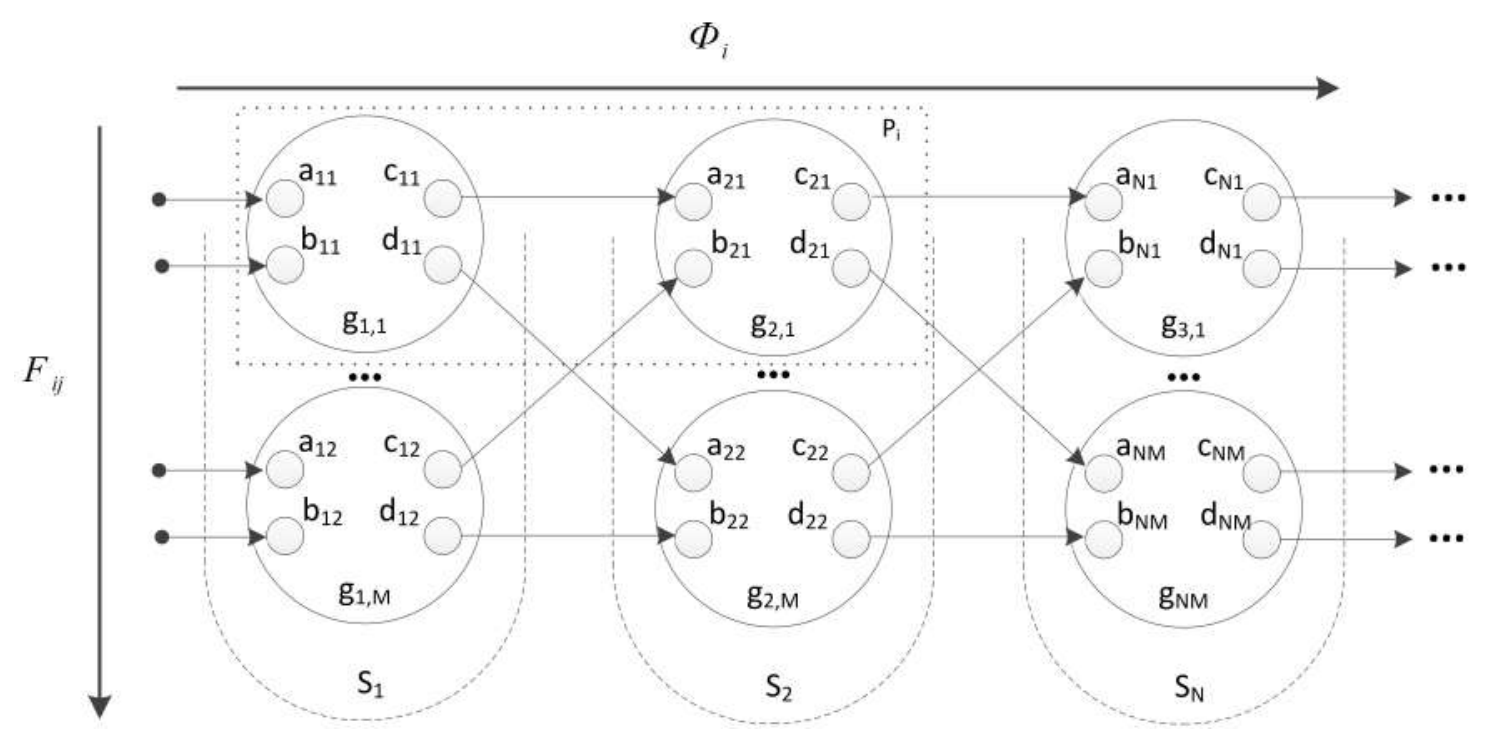

Figure 2. The information graph, its layers $F_{i j}$ and iterations $\Phi_{i}$

A basic subgraph $g_{i j}$ is a minimal indivisible element of a task. When its computing structure is mapped on an RCS, it is completed with functions of reading, writing, and recursion, derived from $F_{i j}$ and $\Phi_{i}$ functions. The obtained indivisible program structure is called a cadr. For all obtained cadrs we specify an order relation, which, together with the von-Neumann determinism, define the execution sequence of cadrs according to their control program.

A basic subgraph is a functionally completed fragment of a TIG. It consists of subgraphs of one or several subtasks. It is possible to map any basic subgraph on an available RCS hardware resource. Completed with the synthesized read/write functions, a basic subgraph provides solution of a task. Within the theory of structural and procedural calculations, basic subgraphs are selected according to available hardware resource. In this case, selection criteria are not formalized; they are determined by the structure of a task, by available resource, and by the developers experience. To select a basic subgraph, the developer analyzes the TIG and looks for frequently used fragments of the TIG which are typical for a certain problem area. Here are the examples of such frequently used fragments:

- addition, multiplication, and division of matrix elements (linear algebra);

- calculations in mesh points (mathematical physics);

- round transformations with logical "AND", "OR", "exclusive OR", and fixed-size data block offset (symbolic processing);

- the discrete fast Fourier transform operation (digital image and signal processing).

Usually, these standard fragments form basic subgraphs of various tasks. We can select basic subgraphs in procedural programs, using descriptions of loops, because fragments with cyclic processing correspond to functional subgraphs, i.e. to calculations with specified scaling functions by layers $F_{i j}$ and by iterations $\Phi_{i}$. Here, the operators of a loop body are a basic subgraph. Information dependencies among operators, and cycle description determine the functions of layers $F_{i j}$ and by iterations $\Phi_{i}$. As a rule, any basic subgraph consists of multiple functional subgraphs, and is a broader concept. However, for some tasks a functional subgraph and a basic one are the same. 


\section{Mapping of Information Graphs on Reconfigurable Computer Systems}

The available hardware resource $A_{R C S}$ defines not only mapping functions $F_{i j}$ and $\Phi_{i}$, but also the calculations of the basic subgraph. That is why, we can represent (1) as

$$
G\left(A_{R C S}\right)=\Phi_{\text {par }} \stackrel{A_{R C S}}{\circ} \Phi_{\text {pipe }}\left(F_{\text {par }} \stackrel{A_{R C S}}{\circ} F_{\text {pipe }}\left(g_{\text {str }} \stackrel{A_{R C S}}{\circ} g_{\text {proc }}\right)\right)
$$

where "par" and "pipe" mean parallel and pipeline execution, respectively; $g_{s t r}$ is the structural form of the basic subgraph; $g_{\text {proc }}$ is the procedural form of the basic subgraph; ${ }^{A_{R C S}}$ is composition of scaling functions, which depends on the available hardware resource $A_{R C S}$.

Using the dependence between the basic subgraph and the available hardware resource 2 , we can describe not only extreme variants of completely structural $\left(g_{s t r}\right)$ and completely procedural $\left(g_{\text {proc }}\right)$ calculations, but other intermediate ones. However, we cannot obtain the structural form $g_{s t r}$ for some tasks due to hardware resource limitations, and the procedural form cannot provide results of adequate accuracy in reasonable time. The examples of such tasks are:

- molecular simulation (docking of inhibitors);

- synthesis of new chemical compounds;

- 3D simulation of spatial physical processes (e.g. tomography of the Earth surface);

- high-resolution simulation of physical processes;

- symbolic processing, etc.

Tasks with variable data flow density 20] belong to this type also. For such tasks, the amount of processed data in various TIG subtasks may differ by 2-4 decimal places, and may depend on input data. For such tasks, basic subgraphs from different layers are significantly non isomorphic. If we try to transform them into isomorphic subgraphs, using the union operation, then we need an inaccessible hardware resource for their structural (or structural-procedural) variant. Hence, we cannot solve these tasks using structural, structural-procedural, or procedural calculations.

If we want to solve these tasks during some reasonable time and using some available hardware resource, it is necessary to reduce the hardware costs for $g_{s t r}$, not using the completely procedural variant $g_{\text {proc }}$, in order to create the basic subgraph within an RCS, and to provide the specified task performance. Here, the task performance is lower than the one for the structural variant $g_{s t r}$, but higher than the one for the procedural variant $g_{\text {proc }}$.

Therefore, we consider a basic subgraph as a scalable, not an atomic object of a task. If we reduce the performance and hardware costs, then it is possible to fulfill all requirements of the task and solve it.

For the first time 20], it was suggested to use performance reduction methods for decreasing of hardware costs in case, when RCS hardware resource is insufficient for even one basic subgraph. The main effect of performance reduction is a linear increase of the task solution time, proportional to the reduction coefficient. The main reduction transformations, which provided balanced scaling of molecular docking tasks in 20], are the following:

- $R^{N}$ - the reduction by number of basic subgraphs. It decreases the number of computing structures, simultaneously mapped on RCS.

- $R^{O p}$ - the reduction by number of computing devices. It decreases the number of concurrently performed operations of the basic subgraph. Similar operations and data of similar 
types are combined in one device. Besides, new connections for operands synchronizations are synthesized.

- $R^{\rho}$ - the reduction by data width. It decreases the number of concurrently processed digits. Absolutely parallel processing of digits in each operand is transformed in partly parallel or sequential processing.

- $R^{S}$ - the reduction by data processing interval. It increases the data processing/supply interval; the hardware costs remain unchanged. This type of reduction is used for matching of data flow with different density among different subtasks or information graph fragments.

- $R^{F r e q}$ - the reduction by clock rate. It decreases the clock rate of a computing structure, which implements some information graph fragment, and matches data flows with different density.

In [20], all reduction transformations were used to reduce hardware costs for solution of some task. However, we can consider the methods of performance reduction as transformations, which provide scaling of a TIG as a computing structure for further mapping on RCS architecture. Moreover, we often use the reduction transformations to get a computing structure from the absolutely parallel form of a task. As a result, the performance of the obtained computing structure is lower, but the structure requires less number of channels and simultaneously operating devices, and/or less data width. That is why we may consider the computing structure of a TIG, mapped on RCS architecture, as performance reduction. Of course, this is true only in case, when RCS hardware resource is insufficient for task solution.

We efficiently use the methods of performance and hardware costs reduction for information graph mapping on RCS architectures. These methods provide automatic (without the programmers instructions) adaptation of applications to various RCS architectures, and solve the problem of application portability.

\section{Methods of Performance and Hardware Costs Reduction}

The task solution performance is a number of computing operations performed per time unit during execution of an application. Let us have a computing structure $F$ with $N_{F}$ basic subgraphs. Each basic subgraph contains $O p_{F}$ computing devices, which process data of a $\rho_{F}$ width. The total number $N C_{F}$ of computing operations, required for processing of a data flow with a length $N$, is

$$
N C_{F}=N \cdot N_{F} \cdot O p_{F} \cdot \rho_{F} .
$$

The task solution time for a computing device with a clock period $\tau=1 /$ Freq and with an interval $S$ is $t=N \cdot S \cdot \tau$. Here, the data processing interval is measured in cycles. Then, the performance of the computing structure $F$ is defined as

$$
\operatorname{Perf} f_{F}=\frac{N C_{F}}{t}=\frac{N \cdot N_{F} \cdot O p_{F} \cdot \rho_{F}}{N \cdot S \cdot \tau}=\frac{N_{F} \cdot O p_{F} \cdot \rho_{F}}{S \cdot \tau}=\frac{N_{F} \cdot O p_{F} \cdot \rho_{F} \cdot F r e q}{S} .
$$

If we carry out the performance reduction with the integer reduction coefficient $R$, then the performance 2 is reduced by $R$ times:

$$
\operatorname{Perf}_{F}(R)=\frac{\operatorname{Perf} F}{R}=\frac{N_{F} \cdot O p_{F} \cdot \rho_{F}}{S \cdot \tau \cdot R}=\frac{N_{F} \cdot O p_{F} \cdot \rho_{F} \cdot F r e q}{S \cdot R} .
$$

The balance of a result computing structure is one of the main and the most important distinction of the performance reduction methods. It means that data flows and hardware costs 
for their switching and synchronization are multiply scaled. Concerning [5, it means that the cofactors of the numerator are reduced by the reduction coefficient $R$ (or by its prime cofactors). According to 5, we can reduce the performance of a task computing structure by:

- decreasing of the number $N_{F}$ of hardware-programmed basic subgraphs in proportion to $R$ (or its prime cofactors). For each mapped BS, the length $N$ of its processed data flow increases. This method is traditional for scalable calculations, performed on RCS and clusters;

- reducing of the number $O p_{F}$ of computing devices in the task basic subgraph [20] in proportion to $R$ (or its prime cofactors). The number of operations, performed by each computing device, and the number of data processing cycles increase. This method is used for RCS;

- decreasing of the processed data width $\rho_{F}$ in proportion to $R$ (or its prime cofactors). The method is used for fixed-point data, and used with restrictions for floating-point data. The number of processing cycles multiply increases. At the same time, the number of data processing channels multiply decreases. This reduction is used in case of lack of input data channels (the most typical case for RCS);

- increasing of the data processing interval $S$;

- decreasing of the clock rate Freq [20].

In the first, second, and third cases, we reduce both the performance and the hardware costs for the computing structure $F$, if the switching and synchronization costs do not exceed the reduced resources. If we use the two last methods, we only reduce the performance of a task or its fragment. In this case, the hardware costs remain unchanged. We can use these methods for matching of data processing rates in different task fragments.

So, we reduce the hardware costs and the number of RCS channels, needed for the computing structure $F$, only if the hardware costs for switching and synchronization do not exceed the reduced resources.

The performance reduction methods without hardware costs reduction are the following:

- the reduction by clock rate;

- the reduction by data processing interval.

A multiple integer, unified for all task fragments automatic performance reduction 5, provides a balanced computing structure. Thus, all task fragments are to be reduced not only with the same reduction coefficient $R$, but the types and coefficients of performed reductions are to be the same. However, for real tasks such requirement is almost impossible.

If we reduce the performance in order to decrease the hardware costs, then all types of reduction transformations are performed in a balanced manner. Here, the reduction coefficient is a positive integer not less than unity. Owing to the reduced computing structure, we can solve the task on lesser hardware resource with longer solution time (in proportion to the reduction coefficient).

In order to describe all reductions of the modifying computing structure, we suggest to use an operation, which rounds rational numbers down to unity 21]. For natural numbers $a \geq 1$ and $b \geq 1$, the operation is defined as

$$
\left\lfloor\frac{a}{b}\right\rfloor_{1}=\left\{\begin{array}{l}
a \operatorname{div} b, a>b \\
1, a \leq b
\end{array}\right.
$$


where div is integer division; \lfloor\rfloor$_{1}$ is similar to the standard floor notation \lfloor\rfloor 21], and it indicates that the result of the "floor" operation is bounded below by unity.

The result of the floor operation \lfloor\rfloor$_{1}$ corresponds to the physical meaning of the parameters that are being reduced, because the number of basic subgraphs, computing devices, and processed digits cannot be less than unity after the reduction. The traditional "floor" operation \lfloor\rfloor has a useful property given in 21]. For the real numbers $m, x$ and the natural number $n$

$$
\left\lfloor\frac{\left\lfloor\frac{x}{m}\right\rfloor}{n}\right\rfloor=\left\lfloor\frac{x}{m \cdot n}\right\rfloor .
$$

Since the set of natural numbers is a subset of the set of real numbers, and function (4) is monotonic and continuous, equality 7 is valid for the proposed function, too. Taking into account the commutative law, we obtain:

$$
\left\lfloor\frac{\left\lfloor\frac{x}{m}\right\rfloor_{1}}{n}\right\rfloor_{1}=\left\lfloor\frac{\left\lfloor\frac{x}{n}\right\rfloor_{1}}{m}\right\rfloor_{1}=\left\lfloor\frac{x}{m \cdot n}\right\rfloor_{1} .
$$

Taking into account 8, we prove the following important theorem, which represents the reduction coefficient as a production of coefficients for the sequential reduction transformation. We denote sequential reduction by $\times$.

For example, the sequential reductions by number of basic subgraphs and by number of computing devices we represent as $R_{n}^{T} \times R_{O p}^{T}=R_{n \cdot m}$.

\section{Theorem 1.}

Sequential T-type reductions $R_{m}^{T}$ and $R_{n}^{T}$ with natural coefficients $m>1$ and $n>1$ are equivalent to the reduction $R_{n \cdot m}^{T}$ of the same type with a coefficient $(m \cdot n)>1$ :

$$
R_{n}^{T} \times R_{m}^{T}=R_{n \cdot m}^{T}
$$

Proof. Let $F$ be a task fragment which contains $N_{F}$ basic subgraphs. Each basic subgraph contains $O p_{F}$ computing devices and processes data with a width $\rho_{F}$. The total amount of calculations $N C_{F}$ in $F$ is

$$
N C_{F}=N_{F} \cdot O p_{F} \cdot \rho_{F} .
$$

Since reduction transformations are independent, then we prove 9 for each type of reduction.

Let us prove condition 9 for the reduction $R_{n}^{N}$ by the number of basic subgraphs with the reduction coefficient $n$. The number of basic subgraphs in $F$ is reduced to $\left\lfloor\frac{N_{F}}{n}\right\rfloor_{1}$, and the total amount of calculations $N C_{n}^{N}$ is:

$$
N C_{n}^{N}=\left\lfloor\frac{N_{F}}{n}\right\rfloor_{1} \cdot O p_{F} \cdot \rho_{F}
$$

The sequential reduction $R_{m}^{N}$ of the same fragment provides $m$-fold decreasing of its number of basic subgraphs. According to 8, we transform [11, and obtain

$$
N C_{n \times m}^{N \times N}=\left\lfloor\frac{\left\lfloor\frac{N_{F}}{n}\right\rfloor_{1}}{m}\right\rfloor_{1} \cdot O p_{F} \cdot \rho_{F}=\left\lfloor\frac{N_{F}}{n \cdot m}\right\rfloor_{1} \cdot O p_{F} \cdot \rho_{F} .
$$

The total amount of calculations 12 , which we obtain as results of the sequential reductions by number of basic subgraphs with the coefficients $n$ and $m$, and as results of the reduction $R_{n \cdot m}^{N}$ 
by number of basic subgraphs with $n \cdot m$ instead of $n$ in 11 , , have the same value. This fact proves Theorem 1. In a similar way, we prove 9] for the reduction by number of computing devices, and for the reduction by data width. As a result, we prove Theorem 1 in general.

Let us formulate several corollaries for application of reduction transformations.

Corollary 1.1 of Theorem 1. Using factorization of performance reduction coefficients, we decrease the number of steps, required for selection of reasonable coefficients for sequential reductions of the same or different types. Besides, for the specified reduction coefficient we choose the best suited type of reduction transformations according to the parameters of a solving task. If the performance reduction coefficient $R$ is a prime number, which exceeds 2 , and if we cannot obtain it by a single reduction, then it is reasonable to perform not an $R$-fold, but an $(R+1)$ fold reduction. In this case, we obtain fully $R$ times lower hardware costs. Since $(R+1)$ is an even composite number, we sequentially perform reduction transformations with reduction coefficients taken from the prime factorization of $(R+1)$.

Corollary 1.2 of Theorem 1. There is no need to return to the initial basic subgraph, when the reduction coefficient multiply increases during sequential reduction of one and the same. If the result of a reduction transformation is a computing structure, which requires additional multiple (not less than twofold) decreasing of hardware costs, and its reduction type permits multiple increasing of its coefficient, then, according to Theorem 1, sequential reduction with no return to the initial basic subgraph lessen the number of steps to get a final reduced structure.

According to Theorem 1, Corollaries 1.1 and 1.2, the total coefficient of sequential reductions equals to a product, but not to an algebraic sum of reduction coefficients. Therefore, it is impossible to get a reduced computing structure with a coefficient from a structure with a coefficient $(n+1)$ using sequential reductions of any type. Let us prove this statement (or Theorem 2) more strictly for a generalized case with a reduction coefficient $(n+x)$.

\section{Theorem 2.}

In the general case, for a basic subgraph reduced with a coefficient n, we cannot obtain a computing structure with a reduction coefficient $(n+x)$ for a prescribed $x \geq 1$, using sequential reductions of a type $T$ with a natural coefficient $k>1$ :

$$
R_{n}^{T 1} \times R_{k}^{T 2} \neq R_{n+x}^{T}
$$

Proof. According to Theorem 1, it is possible to fulfil 13 for reductions of the same type $T$ only if

$$
n \cdot k=n+x
$$

is valid.

Then, we transform 14) and obtain

$$
k=1+\frac{x}{n}
$$

According to Theorem 2, the numbers $n, k$ and $x$ are positive integers. So, we solve 15 for $k$ only when $x$ is integrally divided by $n$, but not $\forall x \geq 1$. This fact proves Theorem 2 .

If we perform reductions of different types, then the similar computing structures from the left and right sides of 13 have the same total amount of operations

$$
N C_{n \times k}^{T 1 \times T 2}=N C_{n+k}^{T}
$$


Therefore,

$$
\left\lfloor\frac{N C}{n \cdot x}\right\rfloor_{1}=\left\lfloor\frac{N C}{n+x}\right\rfloor_{1}
$$

which requires

$$
n \cdot k \geq n+x \quad \text { and } \quad n+x \geq n \cdot k
$$

to be fulfilled.

Both conditions are true only if

$$
n \cdot k=n+x .
$$

So,

$$
k=1+\frac{x}{n} .
$$

Under hypothesis of Theorem $2, n, k$, and $x$ are positive integers. Hence, the solution of 20 for $k$ is possible in the natural domain only when $x$ is integrally divisible by $n$, but not when $\forall x \geq 1$. This conclusion leads to contradiction and, as a result, proves Theorem 2 .

Using Theorem 2, we formulate a corollary which is important for application of a sequence of reduction transformations.

Corollary 2.1 of Theorem 2. For a reduced structure, it is impossible to increase the reduction coefficient by an arbitrary value, performing sequential reductions of any types. Hence, in general case, if additional reduction (of hardware costs) is needed, we return to the initial basic subgraph and perform reduction transformations again with a new (increased) reduction coefficient $R$. As a result, we need more steps to obtain the reduced computing structure.

Let us analyse, how a sequence of reduction transformations of various types influences on a final computing structure.

\section{Theorem 3.}

The superposition of reductions of different types (e.g. a reduction T1 with a coefficient n, and a reduction $T 2$ with a coefficient $m$ ) is commutative. Therefore, if we change the order of reductions of different types for a task fragment, then the result information graph of the fragment remains unchanged:

$$
R_{n}^{T 1} \times R_{m}^{T 2}=R_{m}^{T 2} \times R_{n}^{T 1}
$$

where $T_{1}$ and $T_{2}$ are the types of reduction transformations; $n$ and $m$ are the reduction coefficients.

Proof. Let us prove commutativity of sequential reductions. The first reduction is performed by number of basic subgraphs, and the second one - by number of computing devices:

$$
R_{n}^{N} \times R_{m}^{O p}=R_{m}^{O p} \times R_{n}^{N} .
$$

After the reduction $R_{n}^{N}$, which is performed by number of basic subgraphs and has the reduction coefficient $n$, the total amount of calculations $N C_{n}^{N}$ over binary digit bits is

$$
N C_{n}^{N}=\left\lfloor\frac{N_{F}}{n}\right\rfloor_{1} \cdot O p_{F} \cdot \rho_{F}
$$

Since the number of basic subgraphs and the number of computing devices in each basic subgraph are independent values, then the sequential reduction $R_{m}^{O p}$ by number of computing 
devices with the coefficient $m$ decreases only the number of devices, and the total amount of calculations over binary digit bits is

$$
N C_{n \times m}^{N \times O p}=\left\lfloor\frac{N_{F}}{n}\right\rfloor_{1} \cdot\left\lfloor\frac{O p_{F}}{m}\right\rfloor_{1} \cdot \rho_{F} .
$$

For the right side of 21, the sequential reductions $R_{m}^{O p}$ and $R_{n}^{N}$ lead to the same total amount of calculations over binary digit bits:

$$
N C_{m \times n}^{O p \times N}=\left\lfloor\frac{N_{F}}{n}\right\rfloor_{1} \cdot\left\lfloor\frac{O p_{F}}{m}\right\rfloor_{1} \cdot \rho_{F} .
$$

We prove commutativity for all other possible combinations of sequential reductions in the same way. As a result, this fact proves Theorem 3.

Using Theorem 3, we define a corollary for estimation of the number of reduction steps.

Corollary 3.1 of Theorem 3. If the order of reduction transformations is changed, it is not necessary to return to the initial basic subgraph in order to decrease the number of steps.

\section{Performance Reduction Methods for Information Graphs Mapping on Reconfigurable Architectures}

Taking into account the proved theorems and corollaries, let us formulate the main rules information graph adaptation to RCS architectures.

1) To decrease the number of steps of reduction transformations, it is reasonable to choose coefficients of each type of reduction from the prime factorization of the reduction coefficient.

2) If the number of basic subgraphs in an information graph is more than 1 , then it is reasonable to perform the reduction by number of basic subgraphs as the first step of reduction transformations.

In this case, we linearly decrease the required hardware resource such as the number of FPGA logic cells, and the number of channels for data parallelization.

3) If we perform the reduction by number of computing devices, and by data width to decrease the number of steps of reduction transformations, it is reasonable to perform reductions of each type until the value, specified by reduction criteria, is reached. After that, we perform reduction of another type. Here, the value is chosen according to the cofactors of the reduction coefficient of an information graph.

In this case, we reduce additional overhead of switching hardware.

Owing to the performance reduction methods, which we use for information graphs mapping on RCS architectures, it is possible to divide the set of parallelization variants into several classes that consist of isomorphic computing structures. As a result, we have few variants for analysis.

Let us estimate the number of steps of reduction transformations, which we need to adapt an information graph to a reconfigurable architecture. We consider the most general case, when it is necessary to perform all types of reduction transformations (by number of basic subgraphs, by number of computing devices, and by data width) for reduction of hardware costs.

To define the initial value of a performance reduction coefficient $R$ of a computing structure, we use its approximate value the coefficient of necessary hardware costs reduction $R_{T}$, defined as a proportion of the hardware resource, needed for hardware-programmed information graph, to the available RCS resource $A_{R C S}$. The hardware resource $A_{T}$ for hardware-programmed information graph is equal to the sum of hardware costs of all task subgraphs for each architecture 
component of an FPGA (the number of Look-UP Tables (LUTs), Memory LUTs (MLUTs), FlipFlops (FFs), the number of Digital signal processor blocks (DSPs) and Block RAM (BRAMs)). For an RCS we use the parameters of FPGA chips as follows:

$$
\begin{gathered}
A_{T}=\left\{A_{T}^{L U T}, A_{T}^{M L U T}, A_{T}^{F F}, A_{T}^{D S P}, A_{T}^{B R A M}\right\}, \\
A_{R C S}=\left\{A_{R C S}^{L U T}, A_{R C S}^{M L U T}, A_{R C S}^{F F}, A_{R C S}^{D S P}, A_{R C S}^{B R A M}\right\} .
\end{gathered}
$$

A hardware costs reduction coefficient for each resource is a proportion of the hardware costs, needed for task solution, to the available resource. We select the task hardware costs reduction coefficient as the maximum value among the calculated values:

$$
R_{T}=\operatorname{Max}\left(\frac{A_{T}^{L U T}}{A_{R C S}^{L U T}}, \frac{A_{T}^{M L U T}}{A_{R C S}^{M L U T}}, \frac{A_{T}^{F F}}{A_{R C S}^{F F}}, \frac{A_{T}^{D S P}}{A_{R C S}^{D S P}}, \frac{A_{T}^{B R A M}}{A_{R C S}^{B R A M}}\right) .
$$

The initial value of the performance reduction coefficient $R_{0}$ is equal to the coefficient $R_{T}$, rounded up to the nearest integer: $R_{0}=\left\lceil R_{T}\right\rceil$.

For linear and iterative computing structures, used in tasks of symbolic processing and linear algebra, respectively, the hardware costs reduction coefficient $R_{T}$ and the performance reduction coefficient $R_{0}$ can be the same. In most cases, it turns out that for performance reduction with a coefficient, which is equal to the hardware costs reduction coefficient, it is necessary to increase the performance reduction coefficient even more, due to unforeseen switching costs. Since the overall reduction coefficient is nonadditive for sequential reduction (according to Theorem 2), then it is necessary to increment $R_{0}$ by 1 , and to perform reduction with a new coefficient.

Performance reduction is carried out for the initial value of the performance reduction coefficient $R_{0}>1$, which, according to the fundamental theorem of arithmetic, and to Corollaries 1.1 and 3.1 , is a product of prime cofactors:

$$
R^{0}=\prod_{i} r_{0 i}
$$

To perform reduction transformations, taking into account the task parameters, and the prime factorization of the reduction coefficient $R_{0}$, we represent it as a product of three coefficients of reduction transformations:

$$
R^{0}=R_{0}^{N} \cdot R_{0}^{O p} \cdot R_{0}^{\rho}
$$

If $R_{0}$ is a prime number, we increment it by 1 according to Corollary 1.1.

Since in our case all reductions are performed, then all reduction coefficients $R_{0}^{N}$ (by number of basic subgraphs), $R_{0}^{O p}$ (by number of computing devices) and $R_{0}^{\rho}$ (by data width) exceed unity.

In the first step, it is reasonable to perform the performance reduction by number of basic subgraphs with the coefficient $R_{0}^{N}$. In the second step, we perform the reduction by number of computing devices with the coefficient $R_{0}^{O p}$. The extreme case of subgraph reduction by number of computing devices means sequential execution of its operations as $g_{\text {proc }} 2$ in one device (a processor). If the coefficient $R_{0}^{O p}$ is less than the number of devices in a subgraph, then, according to the type and number of used operations, several variants of computing structures are possible (with the different latency time and data supply interval). The reduced computing structure must provide data equivalency of results. Therefore, each of the considered variants contains devices that perform the operations of the basic subgraph, in order to perform all its operations within the reduced computing structure. 
Let us consider reduction by number of devices for a basic operation of the fast Fourier transform with calculation of coefficients. Its information graph contains 16 operations such as 8 multipliers, 4 adders, and 4 subtractors (see Fig. 3a). Taking into account, that hardwareprogrammed addition and subtraction are identical, we claim that 8 multipliers and 8 adders are enough for the hardware-programmed information graph.

In the case of reduction by number of devices for a basic operation of the fast Fourier transform it is possible to suggest not less than 5 different variants called m-subgraphs. Each $\mathrm{m}$-subgraph is characterized by its own data processing interval and hardware costs:

1. An m-subgraph $\mu_{1}$ (minimal, Fig. 3d) contains not more than one device for each type of the operations of the subgraph. For our example, $\mu_{1}$ contains 2 devices - a multiplier and an adder.

2. An m-subgraph $\mu_{2}$ (multiple) represents a multiple reducing of the number of devices in the subgraph, and is similar to factoring out. Several variants of $\mu_{2}$ are possible, such as 8 devices (4 multipliers, 4 adders, Fig. 3b), 4 devices (2 multipliers, 2 adders, Fig. 3c), and 2 devices (1 multiplier, 1 adder, Fig. 3 d).

3. An m-subgraph $\mu_{3}$ contains all devices from a layer with the maximum total number of operations. If it is necessary, the set of operations is complemented with devices to keep data equivalency. In this case, the layer with the maximum total number of operations is involved entirely, and it is executed during one clock cycle. For our example, $\mu_{3}$ contains 8 devices from the first layer (4 multipliers, 4 adders, Fig. $3 \mathrm{~b}$ ).

4. An m-subgraph $\mu_{4}$ is formed by a layer with the maximum number of operation types. If it is necessary, the layer is complemented with devices to keep data equivalency. For our example, $\mu_{4}$ is similar to $\mu_{3}$. It contains 8 devices from the first layer ( 4 multipliers, 4 adders, Fig. 3b).

5. An m-subgraph $\mu_{5}$ (the improved minimal one) is the minimal $\mu_{1}$ with one supplementary device that performs the most repeated operation of a basic subgraph. For some subgraphs, it provides approximately twofold decrease in the data processing interval for the reduced computing structure. For our example, $\mu_{5}$ contains 3 devices ( 2 multipliers, 1 adder).

We formed the list of m-subgraphs on the base of tasks from such problem domains as digital signal processing, symbolic processing, linear algebra, and molecular docking. It is possible to add to the list some new strategies of m-subgraphs synthesis for tasks from other problem domains. However, the total number of possible strategies hardly ever exceeds 10, because the number of problem domains of RCS application is limited. Here, $\mu_{1}, \mu_{2}$ and $\mu_{5}$ are the most interesting m-subgraphs. The m-subgraph $\mu_{1}$ is the most common variant of basic subgraphs from various problem domains; $\mu_{2}$ is the most acceptable for scaling of computing structures, but not always suitable due to the task structure; $\mu_{5}$ is the most time-optimal, if hardware resource is sufficient for additional hardware-programmed device.

After the reduction by devices, in the third step of transformations, the reduction by data width with the coefficient $R_{0}^{\rho}$ is performed for each synthesized m-subgraph. Here, the number of possible variants of reduction by data width for possible data types does not exceed 2 :

- For the reduction by width of logical and integer data (fixed-point data), the decrease in hardware costs is linearly proportional to the reduction coefficient. Therefore, the reduction is performed with the specified coefficient that does not exceed the width of processing data. 


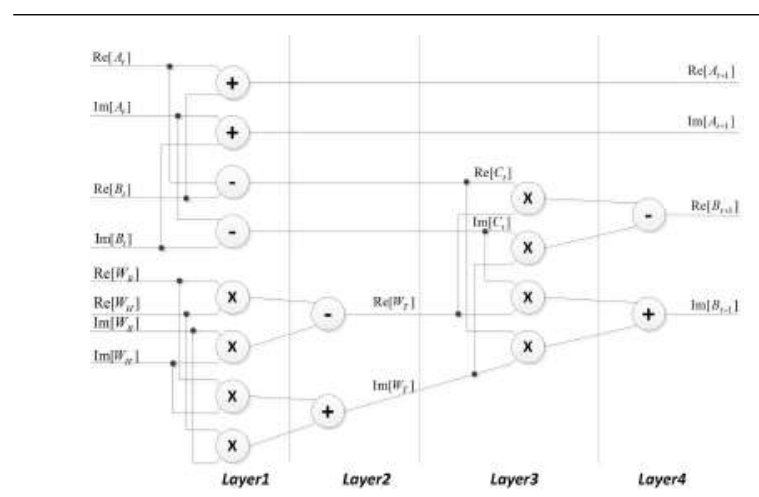

(a) The information graph

of hardware-programmed fast Fourier transform basic operation

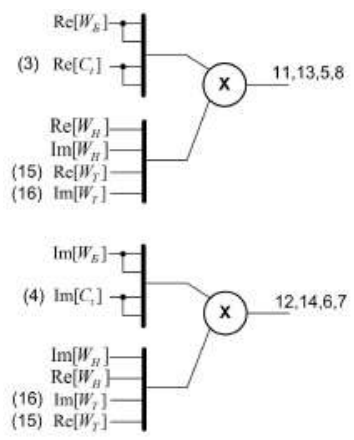

(c) The computational structure

of m-subgraphs $\mu 2-4$ devices, $\mu 3$ and $\mu 4$
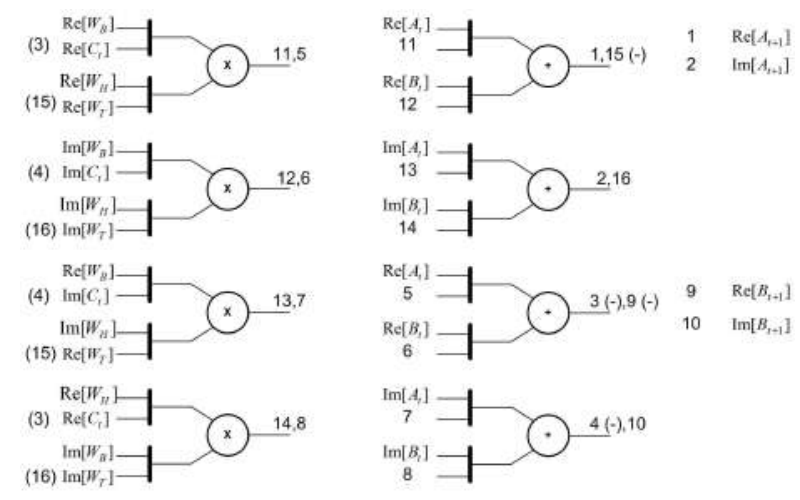

(b) The computational structure of m-subgraphs $\mu 2-8$ devices, $\mu 3$ and $\mu 4$
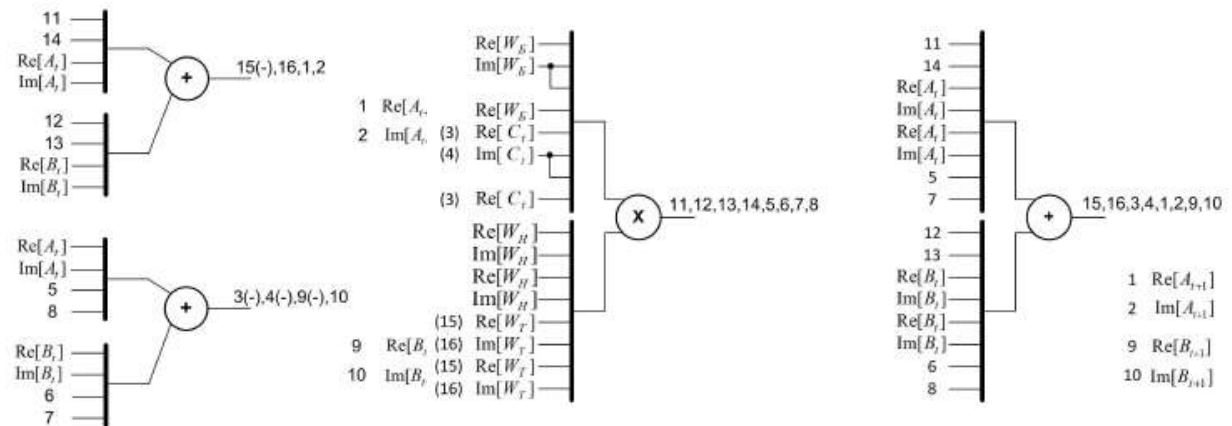

(d) The computational structure of m-subgraphs $\mu 1$ and $\mu 2-2$ devices

Figure 3. The information graph of hardware-programmed fast Fourier transform basic operation and m-subgraphs $\mu_{1}, \mu_{2}, \mu_{3}, \mu_{4}, \mu_{5}$

- If floating-point data are reduced, then it is reasonable to perform 2-fold reduction by data width for 32-digit data, and 2- and 4-fold reduction by data width - for 64-digit data. It is caused by the exponential growth of the overhead expenses for processing of a mantissa and an order of magnitude for other reduction coefficients.

Thus, after reduction by data width, the number of m-subgraph variants is equal to $5 \cdot 2=$ 10. For each variant, it is necessary to analyze the required hardware resource, and the data processing interval, which defines the task solution time. Sometimes, when the hardware costs $A_{T}$ of the reduced task structure exceed the available RCS hardware resource $A_{R C S}$, we perform the additional or fourth step of transformations. Such situation occurs due to additional switching costs, required for the reduction by number of computing devices and for the reduction by floating-point data width, because hardware costs are decreasing non-linearly. For the reduction by number of computing devices, we cannot always calculate the reduction coefficient $R_{0}^{O p}$ before the transformations. Therefore, the coefficients $R_{0}^{O p}$ and $R_{0}^{\rho}$ may demand correction after the reduction.

After all reduction transformations, we evaluate the achieved reducing of hardware costs for task solution. Two variants are possible. We map the reduced computing structure on the available RCS hardware resource, or we additionally reduce hardware costs due to growth of expenses. In the first case, we perform the reduction transformations to map the information graph on the RCS architecture, and it takes 3 steps with analysis of 10 variants. In the second case, we return to the initial information graph (according to Theorem 2), and perform the performance 
reduction (steps 1-3) with the increased coefficient $R_{1}=R_{0}+1$. Or, if it is possible, we perform multiple reductions by one parameter. Obviously, in the second case, the number of analysed variants is duplicated and equal to 20. Even if the task structure consists of several fragments, then the number of variants for justification performed by additional reduction transformations, and by methods of data processing, is few. Here, the additional reduction transformations consist in variation of the clock rate and data processing interval, and data processing can be parallel, pipelined, or can be represented as a macropipeline or a nested pipeline. Practically, the number of different fragments in the most part of tasks does not exceed 3-5; hence, the total number of variants of reduction transformations for such tasks hardly ever exceeds 60 .

When a sequential program for a multiprocessor computer system with distributed memory is parallelized automatically, the compiler evenly distributes all calculations among the nodes without any splitting into several subtasks. It is necessary to analyse data distribution into nodes to avoid data nonlocality that may occur during automatic distribution of calculations disregarding dependencies (Read-After-Write, Write-After-Read, Write-After-Write, ReadAfter-Read) 21, 22] of the source program. So, the parallelizing compiler selects one parameter the parallelizing coefficient according to the number of used multiprocessor computer system nodes, the data spatial locality criterion, and the dependencies of the source program.

Reduction of performance and hardware costs of a RCS is performed with a reduction coefficient, which is the same for all subtasks. As a result, the reduced computing structure is balanced. For an RCS, it is possible to reduce the performance by such parameters as the number of devices, data width, and interval of processing data, unavailable for processor computer systems. For an RCS, in contrast to processor architectures, the overall reduction coefficient for each subtask is represented as a product of reduction coefficients (by number of basic subgraphs, devices, data width and interval). Owing to the fact, that we use a specific combination of reduction coefficients for each subtask, it is possible to take into account parameters of subtasks, choosing the most rational coefficients of reduction transformations, and to decrease the variety of reduced computing structures. Using such approach, we considerably decrease both the number of analyzed variants, and the time of information graph adaptation to the architecture and configuration of the given RCS.

\section{Order of Reduction Transformations For Synthesis of Computing Structures}

We created software tools for application development [24], based on our principles of automatic mapping of information graphs on RCS architectures, and on our performance reduction methods. With the help of the software, any sequential C-program is transformed into the absolutely-parallel information graph form. After that, the information dependencies among the task subgraphs are analyzed, and performance reduction of the subgraphs is performed for further adaptation to the RCS architecture, selected by the user. The methodology of all these transformations is the topic for another paper, and it transcends the scope of this work. Therefore, let us represent the basic rules, which we use for reduction of tasks, containing several subgraphs. To justify the speed of data processing in all subtasks of the information graph, and to select the most rational form of calculations for each subgraph, taking into account computing structures of other subgraphs and the whole task, we use the following order of reduction for computing structure synthesis: 
1. Scaling and performance reduction of the information graph starts from the biggest subgraph. Here, "the biggest subgraph" means the subgraph with the highest hardware costs. The number of memory channels, and the data flow density of the biggest subgraph define all these parameters for all the rest subgraphs.

2. For basic subgraphs partition during analysis of its hardware resource, it is reasonable to compare it with the minimum resource, which is definitely implementable in one FPGA chip. In this case, there is no need to scale the subgraph with the help of the methods of reduction by number of devices, and by data width. If the given minimum resource is sufficient for the subgraph, then the subgraph is hardware-programmed without scaling.

3. The first transformation is decreasing of the number of memory channels. It is performed with the help of the reduction by number of subgraphs for data independent subgraphs. Then, according to the reduction coefficient, all reviewed reduction transformations are performed. Here, we take into account that the order and priority of reduction transformations for different types of tasks can be different.

4. For subgraphs with low weights, it is reasonable to perform hardware implementation. Here, a low weight is not more than a-priori specified value, for example, $5 \%$ from the total hardware costs of the task. If it is necessary to reach the specified reduction coefficient, we use the reduction by data processing interval. Such subgraphs have no considerable influence on exceeding of task hardware resource. Besides, the reduction by number of devices, and by data width can both complicate hardware-programming, and increase hardware costs for switching structure, and, as a result, lead to additional steps of reduction transformations for all task subgraphs.

5. If reduction transformations are the same, but used with different coefficients and in different subtasks, it is necessary to synchronize data flows density (is performed automatically). As a rule, such synchronization leads to additional hardware costs, because hardware programming of synchronization blocks is based on multiplexers/demultiplexers, buffers, internal dual-port memory (BRAM).

6. When we perform the reduction by number of subgraphs, we keep at least one loop structure, because this is the way to decrease the task solution time. Besides, it does not increase the number of distributed memory channels, and it occupies hardware resource, which is available and rather large. If it is impossible, then we program a multipipeline structure. It inevitably contains a feedback, and larger data processing interval; hence, the task solution time grows. Reduction of the data processing interval in such computing structure is possible, if the structure is optimized, i.e. transformed into a nested pipeline or into a macropipeline. In this case, the multipipeline computing structure contains the number of layers equal to the latency of iterative rungs. Then, the computing structure can be reduced to one pipeline, and the feedback sequence is completed with registers. The number of registers is equal to the latency.

7. If the information graph layers have a data dependence, which is possible in the case of functionally irregular graph, then basic subgraphs are reduced to the sequential form.

We experimentally verified all these rules with the help of our compiler prototype and testing tasks of linear algebra, symbolic processing and digital signal processing, such as SLAE solution by the Gaussian method, SLAE solution by the Jacobi method, SLAE solution by lower-upperdecomposition, the basic operation of fast Fourier transform with coefficients calculation. For all these problems, the number of steps of reduction transformations, calculated according to 
the suggested methodology, does not exceed 16. The obtained values of reduction coefficients, numbers of transformation steps, and practical results for the scaled tasks, prove that the reduction transformation methods for automatic creation of parallel RCS applications, reviewed in the paper, are correct and efficient. The efficiency of solutions, created with the suggested methods, is not less than 50-75\% in comparison with optimal solutions, designed by circuit engineers.

\section{Conclusion}

The task information graph, used as the absolutely parallel form of a task for an RCS, provides the maximum performance with the maximum hardware costs. When a task is hardwareprogrammed on an RCS, the user transforms its information graph into a computing structure which provides lower performance and occupies smaller hardware resource. This transformation, decreasing the performance and hardware costs, is performed by reducing the number of subgraphs, computational devices, the processing data width, by increasing the data processing interval, and by reducing the rate. We use performance reduction not only for those tasks, that need more resource, than it is available, but also as a method of mapping (or adaptation) of an information graph to an RCS architecture. Owing to the performance reduction methods for RCS, it is possible to use reduction by number of devices, by data width and interval. This is unachievable for processor computer architectures.

Owing to the proved theorems on reduction transformations, we defined the main principles, and suggested the methodology of information graphs mapping on RCS architectures with the help of the performance reduction methods. Besides, we estimated the number of performed reduction transformations. Performance reduction does not change the total number of variants of a parallel application, but helps us to distribute these variants into several classes for further analysis. It is sufficient to analyze only one variant from each class, not the whole class. The obtained estimation of the number of analyzed variants of the computing structure, synthesized as a result of reduction of performance and hardware costs, is considerably less than the similar indicator for a multiprocessor computer system with distributed memory. We explain it by decomposition of the whole set of variants into topologically isomorphic groups of solutions, performed during reduction. Decrease of the number of analyzed variants to a single computing structure from each class considerably decreases the creation time of a parallel application, adapted to a RCS architecture (or configuration).

Further research will be directed at extension of classes from various problem domains, at mapping of information graphs on RCS architectures with the help of the reviewed methods of automatic reduction of performance and hardware costs.

This paper is distributed under the terms of the Creative Commons Attribution-Non Commercial 3.0 License which permits non-commercial use, reproduction and distribution of the work without further permission provided the original work is properly cited.

\section{References}

1. Voevodin, V.V., Voevodin Vl.V.: Parallel computing. BHV-Petersburg (2002)

2. Palkowski, M., Bielecki, W.: TRACO Parallelizing Compiler. In: Wiliski, A., Fray, I., Peja, J. (eds.) Soft Computing in Computer and Information Science. Advances in Intelligent 
Performance Reduction for Automatic Development of Parallel Applications for...

Systems and Computing, vol. 342, pp. 409-421. Springer, Cham (2015), DOI: 10.1007/9783-319-15147-2_34

3. SAPFOR system. https://www.keldysh.ru/dvm/SAPFOR/, accessed: 2020-05-22

4. Bielecki, W., Palkowski, M.: Perfectly Nested Loop Tiling Transformations Based on the Transitive Closure of the Program Dependence Graph. In: Wiliski, A., Fray, I., Peja, J. (eds) Soft Computing in Computer and Information Science. Advances in Intelligent Systems and Computing, vol. 342, pp. 309-320. Springer, Cham (2015), DOI: 10.1007/978-3-319-15147$2 \_26$

5. Devan, P.S, Kamat, R.K.: A Review - LOOP Dependence Analysis for Parallelizing Compiler. International Journal of Computer Science and Information Technologies 5(3) (2014) https://www.ijcsit.com/docs/Volume\%205/vol5issue03/ijcsit20140503305.pdf, accessed: 2020-05-22.

6. Jensen, N., Karlsson, S.: Improving Loop Dependence Analysis. ACM Transactions on Architecture and Code Optimization 14(3), 1-24 (2017), DOI: 10.1145/3095754

7. Solihin, Y.: Fundamentals of parallel computer architecture: multichip and multicore systems. Chapman and Hall/CRC (2016)

8. Cooper, K.D., Torczon, L.: Engineering a Compiler. Morgan Kaufmann (2005)

9. Kennedy, K., Allen, R.: Optimizing Compilers for Modern Architectures: A Dependencebased Approach. Morgan Kaufmann (2001)

10. Muchnick, S.S.: Advanced Compiler Design and Implementation. Morgan Kaufmann (1997)

11. Levin, I., Dordopulo, A., Fedorov, A., Kalyaev, I.: Reconfigurable computer systems: from the first FPGAs towards liquid cooling systems. Supercomputing Frontiers and Innovations 3(1), 22-40 (2016), DOI: $10.14529 /$ jsfi160102

12. Liu, S., Liu Z., Huang, H.: FPGA implementation of a fast pipeline architecture for JND computation. In: Proceedings of 5th International Congress on Image and Signal Processing, 16-18 Oct. 2012, Chongqing, China. pp. 577-581. IEEE (2012), DOI: $10.1109 /$ CISP.2012.6469995

13. Trimberger, S.M.: Three Ages of FPGAs: A Retrospective on the First Thirty Years of FPGA Technology. Proceedings of the IEEE 103(3), 318-331 (2015), DOI: $10.1109 /$ JPROC.2015.2392104

14. Wahiba, M., Abdellah, S., Aichouche, B.: Implementation of parallel-pipeline H.265 CABAC decoder on FPGA. In: Proceedings of the First International Conference on Embedded \& Distributed Systems, EDiS 2017, 17-18 Dec. 2017, Oran, Algeria. pp. 1-6. IEEE (2017), DOI: $10.1109 /$ EDIS.2017.8284037

15. Khatami, R.I., Ahmadi, M.: High throughput multi pipeline packet classifier on FPGA. In: Proceedings of the 17th CSI International Symposium on Computer Architecture \& Digital Systems, 30-31 Oct. 2013, Tehran, Iran. pp. 137-138. IEEE (2013), DOI: $10.1109 /$ CADS.2013.6714253 
16. Prihozhy, A., Bezati, E., Ab Rahman, A.A., Mattavelli, M.: Synthesis and Optimization of Pipelines for HW Implementations of Dataflow Programs. IEEE Transactions on Computer-Aided Design of Integrated Circuits and Systems 34(10), 1613-1626 (2015), DOI: $10.1109 /$ TCAD.2015.2427278

17. Korcyl, G., Korcyl, P.: Investigating the Dirac Operator Evaluation with FPGAs. Supercomputing Frontiers And Innovations 6(2), 56-63 (2019), DOI: $10.14529 /$ jsfi190204

18. Qu, Y.R., Prasanna, V.K.: High-Performance and Dynamically Updatable Packet Classification Engine on FPGA. IEEE Transactions on Parallel and Distributed Systems 27(1), 197-209 (2016), DOI: 10.1109/TPDS.2015.2389239

19. Kalyaev, I.A., Levin, I.I., Semernikov, E.A., Shmoilov, V.I.: Reconfigurable multipipeline computing structures. Nova Science Publishers, New York, USA (2012)

20. Sorokin, D.A., Dordopulo, A.I., Levin, I.I., Melnikov, A.K.: Solving problems with essentially variable intensity of data flows on reconfigurable computing systems. Bulletin of computer and information technologies 2, 49-56 (2012), DOI: 10.14489/issn.1810-7206

21. Graham, R.L., Knuth, D.E., Patashnik, O.: Concrete Mathematics: A foundation for computer science (2nd ed.). Addison-Wesley Professional (1994)

22. Patterson, D., Hennessy, J.: Computer Architecture: A Quantitative Approach (5th ed.). Morgan Kaufmann (2011)

23. Unnikrishnan P., Shirako J., Barton K., Chatterjee S., Silvera R., Sarkar V.: A Practical Approach to DOACROSS Parallelization. In: Kaklamanis, C., Papatheodorou, T., Spirakis, P.G. (eds) European Conference on Parallel Processing, 27-31 Aug. 2012, Rhodes Island, Greece. Euro-Par 2012 Parallel Processing, Lecture Notes in Computer Science, vol. 7484, pp. 219-231. Springer, Berlin, Heidelberg (2012), DOI: 10.1007/978-3-642-32820$6 \_23$

24. Levin I., Dordopulo, A., Gudkov, V., Gulenok, A., Bovkun A., Yevstafiyev, G., Alekseev, K.: Software Development Tools for FPGA-Based Reconfigurable Systems Programming. In: Voevodin, Vl., Sobolev, S. (eds) Russian Supercomputing Days, 23-24 Sept., Moscow, Russia. Supercomputing, Communications in Computer and Information Science, vol. 1129, pp. 625-640. Springer, Cham (2019), DOI: 10.1007/978-3-030-36592-9_51 